\title{
Reducing anthracycline-induced cardiotoxicity through pharmacogenetics
}

\author{
Erika Scott ${ }^{\ddagger}, 1,2$, Jafar S Hasbullah ${ }^{\ddagger 1,2}$, Colin JD Ross ${ }^{1,2,3}$ \& Bruce C Carleton ${ }^{\star 1,2,4}$ \\ ${ }^{1}$ Department of Medical Genetics, Faculty of Medicine, University of British Columbia, Vancouver, British Columbia, Canada \\ ${ }^{2}$ British Columbia Children's Hospital Research Institute, Vancouver, British Columbia, Canada \\ ${ }^{3}$ Faculty of Pharmaceutical Sciences, University of British Columbia, Vancouver, British Columbia, Canada \\ ${ }^{4}$ Division of Translational Therapeutics, Department of Pediatrics, Faculty of Medicine, University of British Columbia, Vancouver, \\ British Columbia, Canada \\ *Author for correspondence: Tel.: +1 604875 2179; Fax: +1 604875 2494; bcarleton@popi.ubc.ca \\ ${ }^{\ddagger}$ Authors contributed equally
}

\begin{abstract}
"The high inter-individual variability in the development of ACT, even when clinical risk factors are used to determine an individual patient's risk, suggests genetic susceptibility to ACT is also important. The identification of genetic factors for ACT susceptibility provides a means to better predict individual patient risk of ACT from genomic and known clinical risk factors."”
\end{abstract}

First draft submitted: 26 July 2018; Accepted for publication: 30 July 2018; Published online:

14 September 2018

Keywords: adverse drug reactions • anthracycline-induced cardiotoxicity • pharmacogenomics

\section{The need for pharmacogenetics}

Anthracyclines

Anthracycline chemotherapeutics are used to treat a broad spectrum of both childhood and adult cancers [1]. They exert their anticancer effect by intercalating in DNA and inhibiting TOP2A, which is highly expressed in rapidly proliferating cells, to prevent DNA replication and induce apoptosis [2]. These highly effective chemotherapeutic agents have contributed greatly to the $83 \%$ 5-year survival rate of childhood cancers and the $68 \% 5$-year survival rate of adult cancers [3,4].

\section{Anthracycline-induced cardiotoxicity}

Despite their effectiveness, however, the clinical utility of these chemotherapeutics is limited by dose-dependent anthracycline-induced cardiotoxicity (ACT). ACT most commonly presents as subtle changes in cardiac function, but can progress to heart failure in up to $16 \%$ of pediatric patients and $26 \%$ of adult patients $[5,6]$. The risk factors for ACT include pre-existing cardiac disease, age ( $>65$ years old or $<4$ years old), sex (females $>$ males), hypertension, radiation exposure near the heart, cumulative anthracycline dose and anthracycline treatment in combination with other cardiotoxic chemotherapeutic agents, including cyclophosphamide, paclitaxel and trastuzumab [7].

\section{Current strategies to mitigate ACT}

Strategies to mitigate or prevent ACT include continuous infusion of anthracyclines to reduce peak plasma drug level, liposomal encapsulation to alter in vivo distribution and reduce accumulation in cardiomyocytes, and the concurrent use of cardioprotectants such as dexrazoxane [8]. However, in clinical practice, continuous infusion has demonstrated only limited benefit [9], while the high cost of liposomal formulations has hindered their widespread clinical use [8], and the use of dexrazoxane has been hampered by reports of secondary malignancies [10]. Liposomal formulation and continuous infusion strategies are also limited by lack of long-term follow-up data as well as inconclusive evidence that they are effective in pediatric patients [11]. The high inter-individual variability in the development of ACT, even when clinical risk factors are used to determine an individual patient's risk, suggests genetic susceptibility to ACT is also important. The identification of genetic factors for ACT susceptibility provides a means to better predict individual patient risk of ACT from genomic and known clinical risk factors. 
The current state of pharmacogenetics of ACT

Over the past decade, there has been a focused effort by researchers to identify genetic predictors of ACT. There are now over 25 genes harboring genetic variants that have been associated with ACT [11]. We recently published a review that graded each genetic association based on the quality of evidence available and the generalizability to other patient populations [11]. However, many of the genetic associations with ACT require further validation, both in replication cohorts as well as functional and mechanistic studies to confirm the roles of the associated variants in ACT. Many studies were also primarily conducted in pediatric cancer patient populations; thus, the generalizability to adult patient populations remains unknown.

Three of the strongest genetic associations have been incorporated into clinical practice guidelines because they have large effect sizes, have been consistently replicated and show additional evidence for functional involvement in ACT [11]. Two of these variants confer risk: RARG $\mathrm{rs} 2229774\left(\mathrm{OR}[95 \% \mathrm{CI}]=7.0[2.9-17.0], \mathrm{p}=4.1 \times 10^{-8}\right)$ and UGT1A6 rs17863783 (OR [95\% CI] = 4.1 [1.03-16.17], $\mathrm{p}=0.040)$, while the third exhibits protection from ACT: SLC28A3 rs7853758 (OR [95\% CI] $=0.29$ [0.11-0.81], $\mathrm{p}=0.0071)$ [11]. The patients carrying the RARG rs2229774 or UGT1A6 rs17863783 variants are thus considered to be at high risk of ACT while patients carrying the SLC28A3 rs7853758 variant are considered to be at low risk [11]. In the clinical setting, low-risk patients are recommended to receive routine echocardiogram follow-up every 5 years to monitor cardiac function while high-risk patients should have increased echocardiographic monitoring and additional screening for cardiovascular risk factors. High-risk patients are also recommended to receive co-treatment with dexrazoxane or liposomal-encapsulated anthracyclines, or to receive continuous infusion or slower infusion rates of anthracyclines to try to mitigate any cardiotoxicity. These guidelines are currently implemented at British Columbia Children's Hospital and have been used to guide treatment decisions for more than 260 pediatric cancer patients [11]. Studies in pediatric cohorts receiving either doxorubicin or daunorubicin support the conclusions made in these guidelines, and further studies will help expand the current evidence base [11].

\section{Functional relevance of pharmacogenetic findings}

To ensure the relevance of pharmacogenetic findings, variants must be mechanistically validated to show how they contribute to ACT. The roles of some variants associated with ACT have been elucidated, such as that for $R A R G$ rs2229774. One of the proposed pathways for ACT involves anthracycline binding to TOP2B, an isoform primarily expressed in cardiomyocytes over TOP2A, leading to DNA double-stranded breaks, mitochondrial damage and oxidative stress that ultimately result in ACT $[12,13]$. We recently provided further evidence for this mechanism by finding a strong association between $\mathrm{RARG}^{\text {S427L }}$ (rs2229774) and ACT in pediatric cancer patients [14]. RARG normally acts to repress the expression of TOP2B; but when the RARG ${ }^{S 427 \mathrm{~L}}$ variant is present, this repression is reduced, leading to higher expression of TOP2B, anthracycline binding in the heart and ultimately ACT [14]. Functional studies in Top2b-knockout mice and human induced pluripotent stem cell-derived cardiomyocytes also support this proposed mechanism of ACT [12,13].

The roles of other genes and pathways in ACT are currently being explored. SLC28A3, which is marginally expressed in human heart tissue [15], belongs to a class of solute transporter proteins that have been shown to transport anthracyclines in cancer cell lines [16]. Altered expression of the protective SLC28A3 rs7853758 variant may reduce the exposure of cardiomyocytes to anthracyclines [17,18]. UGT1A6 is associated with the glucuronidation pathway in drug metabolism and the UGT1A6*4 haplotype (for which rs17863783 is a tag SNP) is associated with reduced enzyme activity [19]. Consequently, this variant may be linked to reduced elimination of anthracyclines or anthracycline metabolites. Thus, while a more complete picture of the functional roles of RARG, SLC28A3 and UGT1A6 is still developing, there is strong evidence of their involvement in ACT.

These mechanisms and pathways revealed by available genetic evidence can potentially be modulated to prevent ACT. For instance, due to the fact that anthracyclines can bind both TOP2A and TOP2B, strategies to improve the TOP2A specificity of anthracyclines have been proposed to prevent ACT [2]. Other biomarkers, such as TOP2B levels in the blood, are also being explored to predict and prevent ACT [2].

\section{The future of pharmacogenetics for ACT}

Recently, the American Heart Association (AHA) published a scientific statement on cardiovascular disease and breast cancer, highlighting the risk factors and effects of chemotherapeutics on the heart as well as prevention strategies to reduce cardiotoxicity [20]. In this, the AHA failed to mention genetic risk factors as well as the research that has been conducted on the role of genetics in chemotherapy-induced cardiotoxicity. As mentioned in this 
editorial, there has been a joint effort among researchers and clinicians to discover pharmacogenetic variants associated with ACT. As a result, there have now been over 25 genes associated with ACT, and clinical practice guidelines have been developed and implemented for three of these. However, the AHA failing to mention these advancements in their scientific statement highlights the fact that many are still unaware of the clinical utility of pharmacogenetic testing.

Further development of clinical practice guidelines through collaborations among groups with large, diverse study populations comprising both adult and pediatric cohorts, in combination with research on the functional implications of candidate variants, will both expand the pharmacogenomic evidence for ACT and increase awareness of the utility of pharmacogenetic testing. With this in mind, we foresee a future where pharmacogenetics will be used routinely to help reduce the significant healthcare burden associated with adverse events such as ACT and increase the quality of life for many cancer survivors.

\section{Financial \& competing interests disclosure}

Financial support was received from the following government-funded agencies in Canada: Canada Foundation for Innovation (CFI), Canadian Institutes of Health Research (CIHR), Genome Canada, Genome British Columbia and the Provincial Health Services Authority, the University of British Columbia (UBC), and British Columbia Children's Hospital Research Institute. E Scott is currently supported by a CIHR Canada Graduate Scholarship-Doctoral award, a UBC Four-Year Doctoral Fellowship and a CIHR Drug Safety and Effectiveness Cross-Disciplinary Training (DSECT) program award. The authors have no other relevant affiliations or financial involvement with any organization or entity with a financial interest in or financial conflict with the subject matter or materials discussed in the manuscript apart from those disclosed.

No writing assistance was utilized in the production of this manuscript.

\section{References}

1. Smith LA, Cornelius VR, Plummer CJ et al. Cardiotoxicity of anthracycline agents for the treatment of cancer: systematic review and meta-analysis of randomised controlled trials. BMC Cancer 10, 337 (2010).

2. Vejpongsa P, Yeh E. Topoisomerase $2 \beta$ : a promising molecular target for primary prevention of anthracycline-induced cardiotoxicity. Clin. Pharmacol. Ther. 95(1), 45-52 (2014).

3. Ellison LF, Pogany L, Mery LS. Childhood and adolescent cancer survival: a period analysis of data from the Canadian Cancer Registry. Eur. J. Cancer 43(13), 1967-1975 (2007).

4. Siegel RL, Miller KD, Jemal A. Cancer statistics, 2018. CA Cancer J. Clin. 68(1), 7-30 (2018).

5. Swain SM, Whaley FS, Ewer MS. Congestive heart failure in patients treated with doxorubicin. Cancer 97(11), 2869-2879 (2003).

6. Kremer L, Van Dalen E, Offringa M, Voute P. Frequency and risk factors of anthracycline-induced clinical heart failure in children: a systematic review. Ann. Oncol. 13(4), 503-512 (2002).

7. Volkova M, Russell R 3rd. Anthracycline cardiotoxicity: prevalence, pathogenesis and treatment. Curr. Cardiol. Rev. 7(4), 214-220 (2011).

8. Vejpongsa P, Yeh ET. Prevention of anthracycline-induced cardiotoxicity: challenges and opportunities. J. Am. Coll. Cardiol. 64(9), 938-945 (2014).

9. Lipshultz SE, Miller TL, Lipsitz SR et al. Continuous versus bolus infusion of doxorubicin in children with ALL: long-term cardiac outcomes. Pediatrics 130(6), 1003-1011 (2012).

10. Tebbi CK, London WB, Friedman D et al. Dexrazoxane-associated risk for acute myeloid leukemia/myelodysplastic syndrome and other secondary malignancies in pediatric Hodgkin's disease. J. Clin. Oncol. 25(5), 493-500 (2007).

11. Aminkeng F, Ross CJ, Rassekh SR et al. Recommendations for genetic testing to reduce the incidence of anthracycline-induced cardiotoxicity. Br. J. Clin. Pharmacol. 82(3), 683-695 (2016).

12. Maillet A, Tan $\mathrm{K}$, Chai $\mathrm{X}$ et al. Modeling doxorubicin-induced cardiotoxicity in human pluripotent stem cell derived-cardiomyocytes. Sci. Rep. 6, 25333 (2016).

13. Zhang S, Liu X, Bawa-Khalfe T et al. Identification of the molecular basis of doxorubicin-induced cardiotoxicity. Nat. Med. 18(11), 1639-1642 (2012).

14. Aminkeng F, Bhavsar AP, Visscher $\mathrm{H}$ et al. A coding variant in RARG confers susceptibility to anthracycline-induced cardiotoxicity in childhood cancer. Nat. Genet. 47(9), 1079-1084 (2015).

15. Chhibber A, French CE, Yee SW et al. Transcriptomic variation of pharmacogenes in multiple human tissues and lymphoblastoid cell lines. Pharmacogenomics J. 17(2), 137-145 (2017).

16. Nagasawa K, Nagai K, Ohnishi N, Yokoyama T, Fujimoto S. Contribution of specific transport systems to anthracycline transport in tumor and normal cells. Curr. Drug. Metab. 2(4), 355-366 (2001). 
17. Dimas AS, Deutsch S, Stranger BE et al. Common regulatory variation impacts gene expression in a cell type-dependent manner. Science 325(5945), 1246-1250 (2009).

18. Zeller T, Wild P, Szymczak S et al. Genetics and beyond-the transcriptome of human monocytes and disease susceptibility. PLoS ONE 5(5), e10693 (2010).

19. Krishnaswamy S, Hao Q, Al-Rohaimi A et al. UDP glucuronosyltransferase (UGT) 1 A6 pharmacogenetics: II. Functional impact of the three most common nonsynonymous UGT1A6 polymorphisms (S7A, T181A, and R184S). J. Pharmacol. Exp. Ther. 313(3), $1340-1346$ (2005).

20. Mehta LS, Watson KE, Barac A et al. Cardiovascular disease and breast cancer: where these entities intersect: a scientific statement from the American Heart Association. Circulation 137(8), e30-e66 (2018). 